\title{
Enhancement of Satellite Earth Station in Sudan
}

\author{
Mohammed Amer Ahmed, Ibrahim Tarig Mohammed, Hamza Mahdi Albsher
}

\begin{abstract}
- this paper deals with the satellite link budget in 3 different aspects which are:

How to determine the coverage in to percentage form by using Pythagoras theorem in addition of some assumptions, second aspect is the required power toward and from the satellite, and last aspect is how to eliminate the earth station performance in percentage form by using $\mathrm{Eb} / \mathrm{No}$. The research takes 25 locations in the Sudan, gives for each location the specific code. The overall output the OBO ,IBO ,distance ,OFD,C/N,C/Tu ,C/Tco-ch ,C/Ty , Lu ,Ld all are required in order to achieve the 3 aspects. Also enhancing the performance to the optimum in regardless of coverage location is done by controlling in 3 main parameters, this means, the coverage is not a problem in reaching the optimum performance.
\end{abstract}

Keywords - zero performance, Optimum performance, Operational C/T, Receiver G/T, Margin, Frequency.

\section{Introduction}

A satellite link is defined as an Earth station - satellite Earth station connection. The Earth station - satellite segment is called the uplink and the satellite - Earth station segment is called the downlink. The Earth station design consists of the Transmission Link Design, or Link Budget, and the Transmission System Design. The Link Budget establishes the resources needed for a given service to achieve the performance objectives.The Transmission System Design establishes the equipment characteristics necessary to meet the performance objectives for the services to be provided, such as the HPA rated power and the LNA noise temperature. During the analysis, tradeoffs can be made to achieve a balance between cost and performance.

Stations at the edge of the coverage area has poor performance due to fading, noise, low transmitted power and interference signals which related to their location far from the center of the coverage area, in order to enhance these stations, two objectives is set the mean objective is to achieve optimum performance in all sites, the second one, study the effect of the three main parameters and the frequency on the performance and operating EIRP.

In order to overcome this problem we followed several steps,Collection for most parameters dealing with link budget, Analyze all mathematical representations for satellite link using equations, and Obtaining the
Optimum performance, by changing in most Earth station equipment's specially modulation and antennas.

\section{Previous Studies}

In [2] the authors explained the adjustment of earth station antenna towards satellite is caused by decreasing the power link budget in satellite. The quality of transmission signal information could be influenced by changing antenna's direction. This paper discusses the friction features of pointing receiving station antenna that is (Azimuth and Elevation) parameters by using two satellite.[2]

In [3] author's paper has discussed the parameters that affects RF signal while passing through three main parts of the satellite communication. The results of link budget were calculated by Matlab which show the changes in the parameters that causes the weak signal at satellite receiver.[3]

In [4] the authors discussed the calculation of link budget for specific LEO's satellite, considering the signal strength and noise as they are imperative for system design, LEO satellite is used to produce effective signal strength and with less power consumption and minimum loss, to compose data Sheet from link budget that support better communication with effective signal strength.[4]

In [5] The authors talked over the communication link between satellite and earth station is subjected to a lot of impairment such as noise, rain and atmospheric attenuations, as it's susceptible to loss due to antenna misalignment and polarization, this paper presents basics of satellite link budget to adopting project philosophy in future space satellite payload segment promises stable, economical, optimal, broadband and adaptive space operations, in this paper a Gravitational Search Algorithm based on the law of gravity. [5]

In [6] the authors discussed the satellite and earth station communicate with S-band as the quality of communication rely on the performance of ground station which expressed through figure of Merit, the aim of this paper is to investigate the rain attenuation influence on the performance of the respective ground station, rain attenuation depends on geographical location where the satellite ground station is established which in which Pristina, then in different cities the rain attenuation impact on the 
satellite ground station is analyzed through Figure of Merit.[6]

In [7] the authors explained that satellites communication tends to use S-band to communicate with the ground station and communicate at L-band with ecological satellite for search and rescue benefits. The performance of satellite ground station is affected by the antenna noise temperature, which measure of the effective noise integrated over the entire antenna pattern. Heavy rain attenuation is considered assignificant atmospheric factor on determining antenna temperature. In this paper, the calculation and comparison of antenna noise temperature, caused under the worst transmission case, of the assumedsatellite ground station established in different cities. [7]

\section{Scenarios and Results}

This section mainly handles the procedure explored for calculating the coverage area and performance for each earth station and enhancing the satellite earth station performance to the optimum zero percent performance.

\section{The coverage area}

At calculating the coverage area we assumed that the satellite is perpendicular to the center of the earth.

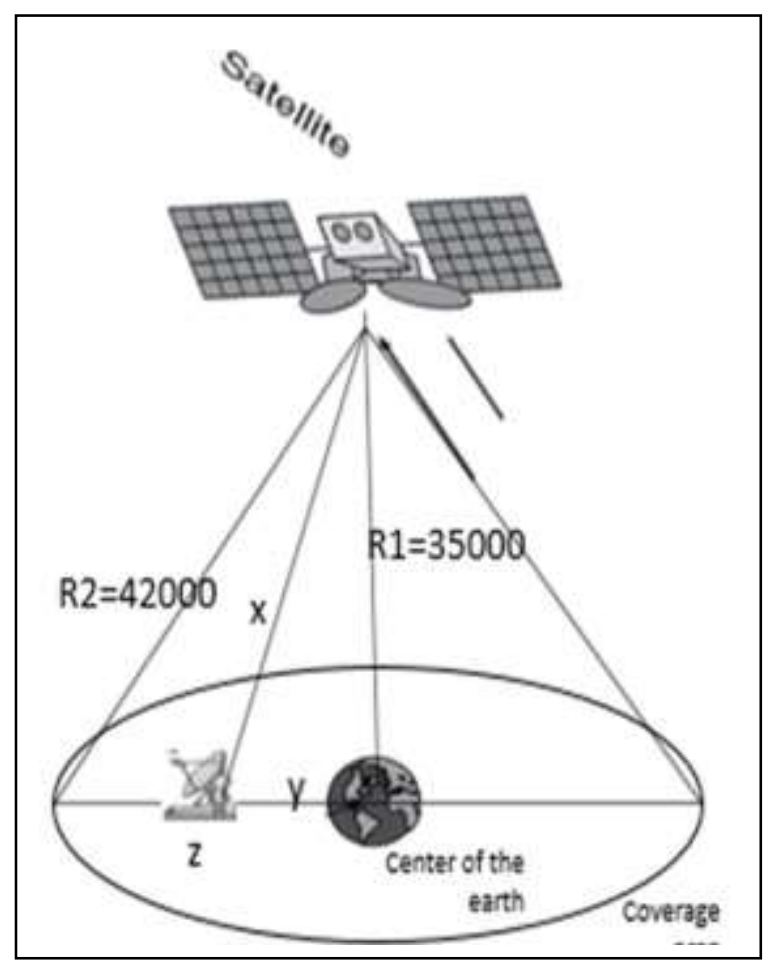

Figure (1) coverage area

Using pythagorth theorem:

$$
\begin{aligned}
Z= & \sqrt{\left(42000^{2}-35000^{2}\right)} \\
X= & R 1^{2}+R 2^{2}+2 R 1 R 2 \cos \emptyset \\
Y= & \sqrt{\left(X^{2}-35000^{2}\right)} \\
& \text { coverage } \\
= & \frac{Z-Y}{Z} \times 100 \%
\end{aligned}
$$

Where:

$\mathrm{R} 1$ : is the distance from satellite to the center of the earth.

R2: radios of geosynchronous orbit.

$\alpha$ : inclination angle of $X$.

The coverage is calculated in percentage form of the distance between the earth station and the edge of the coverage area of the satellite to distance between the center of the earth and the edge of the coverage area of the satellite.

\section{Performance}

As the main objective in this paper is to get the optimum performance for each site in Sudan, we used zero percent concept to simplify understanding the state of performance.

zero performance $=\frac{\text { saturated } C / N-\text { operational } C / N}{\text { saturated } C / N}$

(5)

If the performance was less than zero it meansthat the earth station doesn't operate enough to reach the optimum performance and it need to be enhanced, if more than zero it means that the earth station operate more than enough and need to be reduced.

In this paper all the sites have performance less than zero, enhancement will be implemented on them in order to achieve zero performance. 


\section{The link budget before enhancement}

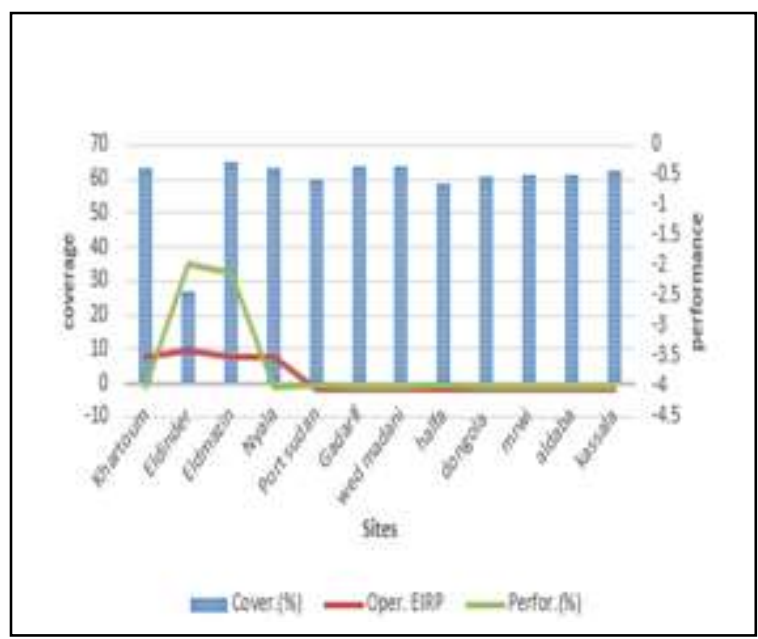

Figure (2) data before enhancing

This graph represent the coverage area, the operating EIRP, and the performance before implement the enhancement on them.

As you see the power range from $-1.689 \mathrm{~dB}$ to 9.760 $\mathrm{dB}$, different coverage for any town from $27.1821 \%$ to $65.0906 \%$, and the performance is less than zero in a range between $-2 \%$ to $-4 \%$ which means it operate less than the optimum performance and it needs to be enhanced.

\section{The link budget after enhancement}

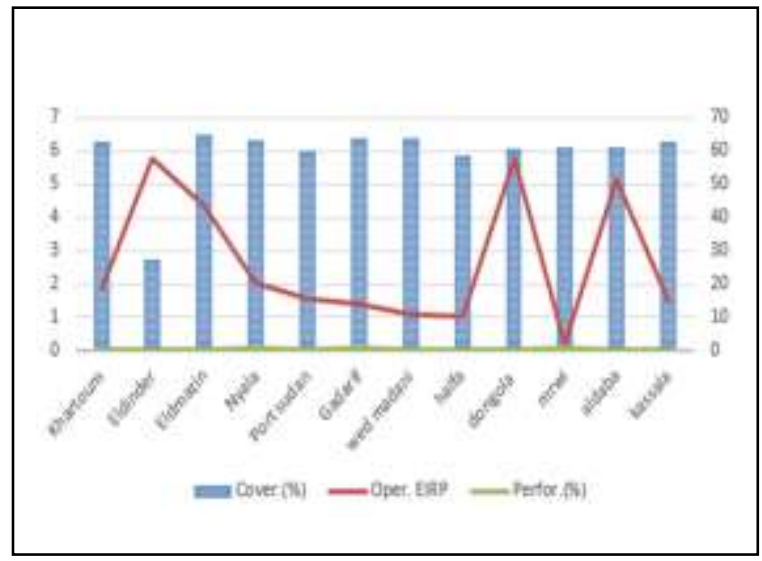

Figure (3) data after enhancing

This graph represent the coverage area, the operating EIRP, and the performance after implementing the enhancement on them.

As you see the power controlled to be less than $7 \mathrm{~dB}$ ranged between $0.17781 \mathrm{~dB}$ and $5.773915 \mathrm{~dB}$, and the performance optimized to zero performance in all sites regard less their coverage areas.

\section{Enhancement effect}

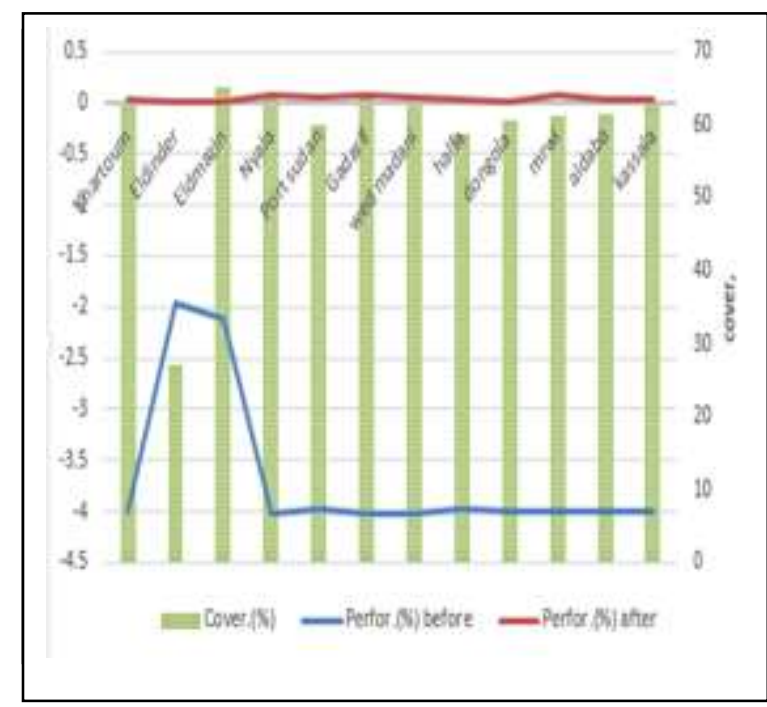

Figure (4) comparison of performance before and after the enhancement

As you see after zero enhancing the line of performance moved up from all poor quantities to zero performance in all sites either it located on the center of the coverage area or on the edge of the coverage area.

\section{Zero Enhancement}

By zero enhancing we mean that manipulating the link budget until performance reach zero performance, here some examples how we achieved zero performance in different sites:

\section{Khartoum:}

Operational $\mathrm{C} / \mathrm{T}$ is changed to $-155.2 \mathrm{dBw} / \mathrm{aK}$ instead of $-157.2 \mathrm{dBw} / \mathrm{nK}$ to increase the performance. The margin is changed in transmitter to $-5.6 \mathrm{~dB}$ instead of $1.5 \mathrm{~dB}$ and in receiver to $8 \mathrm{~dB}$ instead of $3.5 \mathrm{~dB}$.

\section{Eldmazin:}

Receiver G/T decreased to $30.3 \mathrm{~dB} / \mathrm{IK}$ instead of $36.4 \mathrm{~dB} / \Perp \mathrm{K}$ to achieve performance and power 0.000521 and $4.375 \mathrm{~dB}$ instead of -2.1044 and $7.6617 \mathrm{~dB}$ respectively.

\section{Nyala:}

Operational $\mathrm{C} / \mathrm{T}$ is changed to $-155.25 \mathrm{dBw} / \mathrm{K}$ instead of $-157.2 \mathrm{dBw} / \mathrm{K}$, receiver $\mathrm{G} / \mathrm{T}$ increased up to $37 \mathrm{~dB} / \mathrm{K}$ instead of $36.4 \mathrm{~dB} / \mathrm{K}$, the margin is changed in transmitter to $-6 \mathrm{~dB}$ instead of $1.5 \mathrm{~dB}$ and in receiver to $9 \mathrm{~dB}$ instead $3.5 \mathrm{~dB}$, all these changing achieved the both, performance and power which are 0.06690 and $2.0177 \mathrm{~dB}$ instead of-4.0133 and 7.6817 $\mathrm{dB}$ respectively. 


\section{Gadarif:}

Operational $\mathrm{C} / \mathrm{T}$ is changed to $-153.5 \mathrm{dBw} / \mathrm{0K}$ instead of $-157.2 \mathrm{dBw} / \mathrm{0K}$ to increase the performance from 4.01549 to 0.085457 with small change in receiver $\mathrm{G} / \mathrm{T}$ and receiver margin.

And so on...

We can do a huge change in the performance by small change in even (Operational $\mathrm{C} / \mathrm{T}$, margin, and receiver $\mathrm{G} / \mathrm{T}$ ) and control the frequency inside the band to control power consumption, the other parameters just to help for $100 \%$ adjacent.

The effect of the three main parameters and the frequency on the performance and operational EIRP:

\section{Operational $\mathrm{C} / \mathrm{T}$ effect}

Controlling in the operational $\mathrm{C} / \mathrm{T}$ and kept the resting parameters constant, I achieve the optimum performance at $\mathrm{C} / \mathrm{T}$ equal to $-153.8 \mathrm{dBw} / \mathrm{K}$ for Damazin as receiving site and equal to $154.1 \mathrm{dBw} / \mathrm{K}$ for the El-dinder as receiving site, this variation in the $\mathrm{C} / \mathrm{T}$ between the two sites came due their coverage, but they have the same performance although their different coverage. Putting in consideration, the margins are same in all trials which are $3.5 \mathrm{~dB}$, we found that increasing the operational $\mathrm{C} / \mathrm{T}$ Increases the performance from 4.024014 in Damazin and from -3.6689484 in Eldinder, both to the achieved optimum performance. And increases Oper.EIRP from $-1.72 \mathrm{~dB}$ to $1.67 \mathrm{~dB}$ in Damazin and -0.32dB to $2.77 \mathrm{~dB}$ in El-dinder.

\section{Receiver G/T effect}

Controlling in the $(\mathrm{G} / \mathrm{T}) \mathrm{RX}$. and kept the resting parameters constant, I achieve performance close to optimum performance equal to -1.1 at $\mathrm{G} / \mathrm{T}$ equal to $10 \mathrm{~dB} / \mathrm{K}$ for Damazin as receiving site and equal to $15 \mathrm{~dB} / \mathrm{K}$ for EL-dinder as receiving site, this variation in the $\mathrm{G} / \mathrm{T}$ between the two sites came due their coverage, but they have the same performance although their different coverage. Putting in consideration, the margins are same in all trials which is $3.5 \mathrm{~dB}$, we found that decreasing the Receiving (G/T). Increases the performance from 4.024014 in Damazin and from -3.6689484 in Eldinder, both to the achieved -1.1 performance, and increases the Oper.EIRP from $1.72 \mathrm{~dB}$ to $-1.1 \mathrm{~dB}$ in Damazin and from $-0.32 \mathrm{~dB}$ to $21.07 \mathrm{~dB}$ in El-dinder. The effect of receiver G/T couldn't achieve the zero performance, but with small change in any other parameter could achieve it, we only focusing in the effect of the parameter not the zero performance.

\section{Receiving Margin Effect}

Controlling in the receiving margin. and kept the resting parameters constant, I achieve performance close to optimum performance equal to -1.1 at margin is $5 \mathrm{~dB}$ for Damazin as receiving site and is $20 \mathrm{~dB}$ for the El-dinder as receiving site, this variation in the marginbetween the two sites came due their coverage, but they have the same performance although their different coverage. Putting in consideration, the $\mathrm{C} / \mathrm{T}$ for the both sites is same in all trials which is $-157.2 \mathrm{dBw} / \mathrm{K}$, we found that increasing the Receiving margin. Increases the performance from -4.024014 in Damazin and from 3.6689484 in El-dinder, both to the achieved -1.11 performance, and increases the Oper.EIRP from-1.72 $\mathrm{dB}$ to $19.77 \mathrm{~dB}$ in Damazin and from $-0.32 \mathrm{~dB}$ to $16.17 \mathrm{~dB}$.

The effect of receiving margin couldn't achieve the zero performance also.

\section{Iv. Frequency effect}

Controlling in the frequency, by increase it up to $10 \mathrm{GHz}$ instead of $6.2 \mathrm{GHZ}$, and kept the resting parameters constant, putting in consideration, the $\mathrm{C} / \mathrm{T}$ for the both sites is same in all trials which is -157.2 $\mathrm{dBw} / \mathrm{K}$, we found that increasing the frequency.

Doesn't affect in performance at all, and increases the Oper.EIRP from-1.72 $\mathrm{dB}$ to $2.427738 \mathrm{~dB}$ in Damazin and from $-0.32 \mathrm{~dB}$ to $3.826081 \mathrm{~dB}$ in Eldinder.

\section{v. conclusion}

The coverage and the power required toward satellite has been calculated, optimum performance were achieved in different sites in Sudan regard less their location on the coverage area, reach the optimum performance by controlling in different parameters individually or in combine form.

\section{Acknowledgment}

In the name of Allah the most graceful the most merciful

Firstly, I would like to thank God for giving us the strength to finish this accomplishment.

Secondly, I would like to express my appreciation and gratitude to our families for inspiring us and their support.

Thirdly, I would like to thank my teachers in this faculty. They helped us out and provided us with knowledge needed to face our career life. 
Proc. of the Fifth Intl. Conf. Advances in Computing, Communication and Information Technology- CCIT 2017 Copyright (C) Institute of Research Engineers and Doctors, USA .All rights reserved.

ISBN: 978-1-63248-131-3 doi: 10.15224/ 978-1-63248-131-3-23

We would like to dedicate this work to all our families and friends and everyone that helped us, supported us or been there for us thanks everybody.

And a special dedication of course goes back to the father and boss dr.atif Osman Bakhiet may Allah bless him.

\section{References}

[1] I. A. a. Program, Earth Station Technology, June 1999.

[2] P. WahyuPamungkas, Analysing influence of earth station antenna pointing to receiving signal parameter at down link power budget, January, 2009.

[3] A. I. K. G. A. Q. Adil Hussein M. Al-Dalowi, Satellite Link Budget Calculator by Using Matlab/GUI, March, 2017.

[4] D. K. M. S. D. R. P. A. SnehasisDey, An Approach to calculate the Performance and Link Budget of LEO Satellite (Iridium) For Communication Operated at frequency Range (1650-1550) MHz, November, 2014.

[5] C. s. 1. b. o. u. g. s. algorithm, S. Berrezzoug, F. T. Bendimerad, A. Boudjemai, May, 2015.

[6] S. CAKAJ, Rain Attenuation Impact on Performance of Satellite Ground Stations for Low Earth Orbiting (LEO) Satellites in Europe, September, 2009.

[7] B. K. I. E. O. S. ShkelzenCakajKamo, Antenna Noise Temperature for Low Earth Orbiting Satellite Ground Stations at L and S Band, 2011.

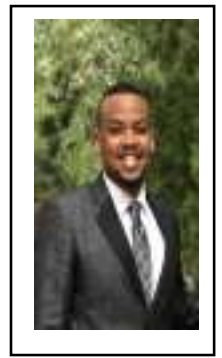

Mohammed Amer Ahmed

Bachelor of Electronics-

communication Engineering

University of Medical Sciences

and Technology, Khartoum-

Sudan

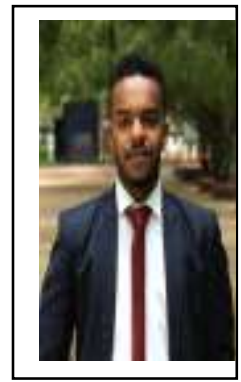

Hamza Mahdi Albsher

Bachelor of Electronics-

communication Engineering

University of Medical Sciences

and Technology, Khartoum-

Sudan

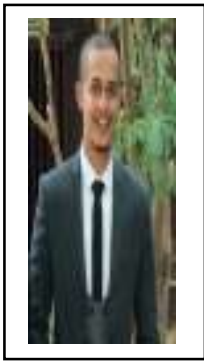

Ibrahim Tarig Mohamed Bachelor of Electronicscommunication Engineering University of Medical Sciences and Technology, KhartoumSudan 